\title{
miR-200c-3p upregulation and ACE2 downregulation via bacterial LPS and LTA as interesting aspects for COVID-19 treatment and immunity
}

\author{
Saber Soltani ${ }^{1,2} \mathbb{D} \cdot$ Milad Zandi $^{1,2}$ \\ Received: 14 March 2021 / Accepted: 24 April 2021 / Published online: 3 May 2021 \\ (c) The Author(s), under exclusive licence to Springer Nature B.V. 2021
}

\section{Commentary}

The Severe acute respiratory syndrome coronavirus 2 (SARS-CoV-2) outbreak was occurred in December 2019 [1]. Coronavirus disease 2019 (COVID-19) was considered a pandemic by the WHO on 12 March 2020. So far, many attempts have been made to design effective antiviral agents and vaccines against SARS-CoV-2. Current studies discussed ACE2, which plays an essential role in the pathophysiology of COVID-19 as a virus receptor [2]. According to studies finding, respiratory microbiota has been considered a notable factor in viral infections [3]. The human respiratory microbiota carries a wide range of gram-positive and gram-negative bacterial cells as the main composition, with different roles in physiological conditions and respiratory diseases [4].

Recent studies have suggested the role of respiratory microbiota in COVID-19 [5]. This commentary's hypothesis is based on the cellular function of gram-positive and gram-negative respiratory bacteria as a co-factor to prevent the expression of ACE2 and thus inhibit SARS-CoV-2. Lipopolysaccharide (LPS) is the outer membrane component in gram-negative bacteria [6]. Also, lipoteichoic acid (LTA) is the primary cell wall constituent in gram-positive bacteria [6]. Bacterial LPS and LTA can be involved in various cellular signaling mechanisms and pathways, including microRNA expression [7]. MicroRNAs are the small non-coding RNAs that impact viral respiratory infections pathogenesis [8]. Besides, the role of microRNAs as therapeutic agents and vaccine design has been discussed [8].

Saber Soltani

Sabersoltani71@gmail.com; s-soltani@razi.tums.ac.ir

1 Department of Virology, School of Public Health, Tehran University of Medical Sciences, Tehran, Iran

2 Research Center for Clinical Virology, Tehran University of Medical Sciences, Tehran, Iran
According to evidence, LPS activates NF- $\kappa \mathrm{B}$ via TLR4 and LTA via TLR2 [7]. On the other hand, activation of the NF- $\kappa B$ pathway increases the expression of miR-200c-3p, which is an important factor in ARDS [7]. Increased expression of miR-200c-3p has been observed to decrease ACE2 expression [7]. It should be noted that low expression of ACE2 in the lungs and the upper respiratory tract in some COVID-19 cases may be associated with a reduction in disease severity [9]. Therefore, it is hypothesized that bacterial LPS and LTA can reduce the expression of ACE2 in the lungs of COVID-19 patients through upregulation of miR-200c-3p.

Murine models of SARS-CoV have confirmed that the virus may stimulate TNF- $\alpha$ and IL- 6 through the NF- $\kappa B$ pathway[10]. Shaath et al. reported, NF-кB was activated in B.A.L. cells of severe COVID-19 [11]. This issue would clarify the high levels of cytokine responses, leading to inflammation and cytokines storm in COVID-19 patients. The cytokines storm in COVID-19 could result in ARDS and multi-organ dysfunction. This aspect could help in the design of effective vaccine and therapeutic approaches [10, 12]. Respiratory bacterial can also develop NF- $\kappa B$ activation, which induces a pro-inflammatory response in epithelial cells $[13,14]$. The human microbiota can be altered after SARS-CoV-2 infection [15]. It is a hypothesis that these microbiota changes can illustrate the cytokines storm progression in COVID-19. Therapy using NF- $\kappa B$ inhibitor drugs leads to decreased inflammation and lung injury in SARS-CoV-infection models [16]. The differential miRNA expression in COVID-19 patients may regulate the inflammatory responses during infection [17]. Also, Some miRNAs can target ACE2 [18]. The "microRNA targeting" as anti-inflammatory agents should be carefully considered. It is well known that microRNAs are strongly involved in cytokines and chemokines expression, leading to cytokines storm in COVID-19 [19].

In conclusion, the composition of bacterial respiratory microbiota can be an indicator of COVID-19 severity. Future 
clinical trials are needed to investigate the role of probiotics containing LPS and LTA in affect on COVID-19 symptoms by upregulation miR-200c-3p levels and downregulation ACE2 levels in COVID-19 patients. Future studies in the gene therapy field could also directly investigate the role of miR-200c-3p as a biomarker in reducing ACE2 expression and COVID-19 severity to provide immunity against SARS-CoV-2.

Authors contribution SS: literature search, writing, design, editing, and final proof. MZ: Editing and final proof.

\section{Declarations}

Conflict of interest The authors have no conflict of interest to declare.

\section{References}

1. Huang C et al (2020) Clinical features of patients infected with 2019 novel coronavirus in Wuhan, China. Lancet 395(10223):497-506

2. Li Y et al (2020) Physiological and pathological regulation of ACE2, the SARS-CoV-2 receptor. Pharmacol Res 157:104833

3. Pichon M, Lina B, Josset LJV (2017) Impact of the respiratory microbiome on host responses to respiratory viral infection. Vaccines(Basel) 5(4):40

4. Dickson RP, Erb-Downward JR, Huffnagle GB (2013) The role of the bacterial microbiome in lung disease. Expert Rev Respir Med 7(3):245-257

5. Khatiwada S, Subedi AJ (2020) Lung microbiome and coronavirus disease 2019 (COVID-19): possible link and implications. Hum Microb J. 17:100073

6. Takeuchi O et al (1999) Differential roles of TLR2 and TLR4 in recognition of gram-negative and gram-positive bacterial cell wall components. Immunity 11(4):443-451
7. Liu Q et al (2017) miRNA-200c-3p is crucial in acute respiratory distress syndrome. Cell Discovery 3(1):1-17

8. Leon-Icaza SA, Zeng M, Rosas-Taraco AGJE (2019) microRNAs in viral acute respiratory infections: immune regulation, biomarkers, therapy, and vaccines. ExRNA 1(1):1-7

9. Yang C, Li Y, Xiao S-YJE (2020) Differential expression of ACE2 in the respiratory tracts and its relationship to COVID-19 pathogenesis. EBio Med. https://doi.org/10.1016/j.ebiom.2020.103004

10. Wang $\mathrm{W}$ et al (2007) Up-regulation of IL- 6 and TNF- $\alpha$ induced by SARS-coronavirus spike protein in murine macrophages via NF- $\kappa$ B pathway. Virus Res 128(1-2):1-8

11. Shaath $\mathrm{H}$ et al (2020) Single-cell transcriptome analysis highlights a role for neutrophils and inflammatory macrophages in the pathogenesis of severe COVID-19. Cell 9(11):2374

12. Pearce L, Davidson SM, Yellon DM (2020) The cytokine storm of COVID-19: a spotlight on prevention and protection. Expert Opin Ther Target. 24(8):723-730

13. Stavropoulou E et al (2021) Unraveling the interconnection patterns across lung microbiome, respiratory diseases, and COVID19. Front Cell Infect Microb 10:892

14. Medina E, Anders D, Chhatwal GS (2002) Induction of NF-kappaB nuclear translocation in human respiratory epithelial cells by group A streptococci. Microb Pathag. 33(6):307-313

15. Soltani $\mathrm{S}$ et al (2021) The role of bacterial and fungal human respiratory microbiota in COVID-19 patients. Biomed Res Int. https://doi.org/10.1155/2021/6670798

16. Vitiello $\mathrm{M}$ et al (2012) NF- $\mathrm{\kappa B}$ as a potential therapeutic target in microbial diseases. Mol Biosyst 8(4):1108-1120

17. Li C et al (2020) Differential microRNA expression in the peripheral blood from human patients with COVID-19. J Clin Lab Anal 34(10): 23590

18. Hum C et al (2021) MicroRNA mimics or inhibitors as antiviral therapeutic approaches against COVID-19. Drugs 81:517-531

19. Gasparello J, Finotti A, Gambari R (2021) Tackling the COVID19 "cytokine storm" with microRNA mimics directly targeting the 3'UTR of pro-inflammatory mRNAs. Med Hypothesis 146:110415

Publisher's Note Springer Nature remains neutral with regard to jurisdictional claims in published maps and institutional affiliations. 